radio position; the arches are composed of systems of sharply defined filaments. The general distribution of the nebulosity in relation to the probable errors of the radio source is shown in Fig. 3.

The system is strikingly similar both in shape and structure to the larger double-loop system of $N G C$ 6960 and $N G C 6992$ in Cygnus, and although no optical spectra of the Gemini loops are yet available to confirm this similarity, both a search for radio emission from the Cygnus loops and further optical studies of galactic nebulæ of this type might yield additional information about the nature of the galactic radio sources.

The radio observations were carried out as part of a programme of research supported in the Cavendish Laboratory by the Department of Scientific and Industrial Research, to which one of us (J.E. B.) is indebted for a maintenance allowance. We are also grateful to Mr. P. A. O'Brien for permission to use his unpublished results.

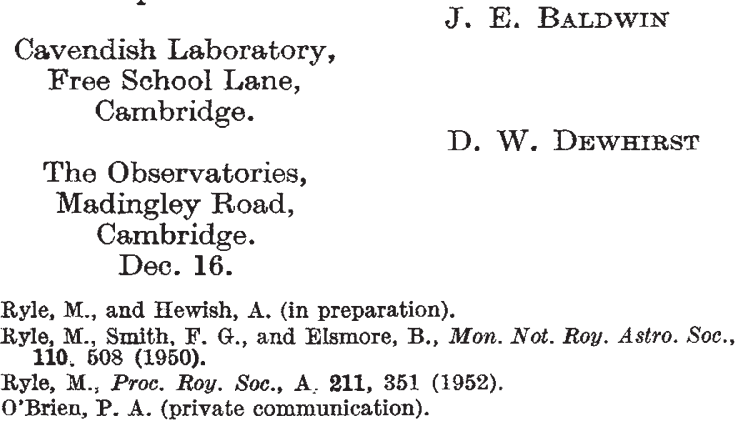

\section{Recombination in the F-Layers}

Some years ago, Bates and Massey ${ }^{1}$, after showing the influence of negative ion reactions to be unimportant, suggested that dissociative recombination is the fundamental process responsible for removing electrons from the $E$-layer ; and later they ${ }^{2}$ suggested that the mechanism might also be effective in the $D$-layer. Recent experimental ${ }^{3}$ and theoretical ${ }^{4}$ work indicates that the recombination coefficient is probably of the required high magnitude. The $F$-layers are at least partially, and perhaps mainly, due to the photo-ionization of atomic oxygen, so it is not immediately obvious that dissociative recombination can be operative in them. However, Bates and Massey tentatively proposed that the necessary molecular ions are formed through charge transfer collisions :

$$
\mathrm{O}^{+}+X Y \rightarrow \mathrm{O}+X Y^{+}
$$

$X Y$ being some unspecified atmospheric constituent.

In a communication in Nature, Banerji $\mathrm{i}^{5}$ has sought to identify $X Y$ as metastable nitrogen, which he supposes reacts through :

$$
\begin{aligned}
\left.\mathrm{O}^{+}{ }^{\left({ }^{4} S\right.}\right)+\mathrm{N}_{2}\left(A^{3} \Sigma_{u}\right) \rightarrow \mathrm{N}_{2}+\left(X^{2} \Sigma_{g}\right) & +\mathrm{O}\left({ }^{1} S\right)+ \\
& \sim 0.01 \mathrm{eV} .
\end{aligned}
$$

It may be noted that this process is rather more complicated than ordinary charge transfer, since a system in the ${ }^{1} S$ state cannot be obtained by merely adding an electron to a system in the ${ }^{4} S$ state. Arbitrarily assuming the rate coefficient to be as grest as $10^{-11} \mathrm{~cm}^{3} / \mathrm{sec}$. , Banerji finds the required concentration $\left[\mathrm{N}_{2}\left(A^{3} \Sigma_{u}\right)\right]$ is of order $10^{7} / \mathrm{cm}^{3}$, which he points out is some $2 \times 10^{-2}$ times the concentration of normal nitrogen molecules $\left[\mathrm{N}_{2}\left(X^{1} \Sigma_{g}\right)\right]$.
He claims that this high degree of excitation could be maintained during the day by the action of solar radiation :

$$
\mathrm{N}_{2}\left(X^{1} \Sigma_{g}\right)+h \nu \rightarrow \mathrm{N}_{2}\left(A^{3} \Sigma_{u}\right)
$$

In fact, however, we have that $\left[\mathrm{N}_{2}\left(A^{3} \Sigma_{u}\right)\right] /\left[\mathrm{N}_{2}\left(X^{1} \Sigma_{g}\right)\right]$ is given by :

$$
F^{\prime}=\left(r \omega_{A} / \omega_{X}\right) \Omega \exp .(-h \nu / k T),
$$

where $r$ is a number, smaller than unity, which allows for collisional deactivation and for the difference between the Franck-Condon factors of the upward and downward transitions; the $\omega$ 's are the statistical weights of the states indicated; $\Omega$ is the solar dilution factor ; and $T$ is the effective temperature of the sun at the frequency $v$ concerned in the transition. Neglecting $r$ and taking $T$ to be $6,000^{\circ} \mathrm{K}$. (which rocket measurements show is a gross overestimate ${ }^{6}$ ), we find on substituting in (4) that $F$ is $10^{-10}$ or less, which is far below the $2 \times 10^{-2}$ required. Quite apart from this, it may be mentioned that if $\left[\mathrm{N}_{2}\left(A^{3} \Sigma_{u}\right)\right]$ were as large as proposed, the First Positive System would appear prominently in the twilight air-glow due to resonance scattering?. It is concluded, therefore, that the hypothesis under discussion is unacceptable.

I am indebted to Prof. D. R. Bates for helpful discussions.

Department of Applied Mathematics,

A. L. STewart

The Queen's University,

Belfast.

Dec. 2.

1 Bates, D. R., and Massey, H. S. W., Proc. Roy. Soc., A, 192, 1 (1947).

${ }^{2}$ Bates, D. R., and Massey, H. S. W., J. Terr. and Atmos. Elect., 2, 253 (1952).

${ }^{3}$ Biondi, A., and Brown, S. C., 'Phys. Rev., 76, 1697 (1949).

4 Bates. D. R., Phys. Rev., 78, 492 (1950).

${ }^{5}$ Banerji, R. B., Nature, 172, 953 (1953)

- Johnson, F. S., Purcell, J. P., Tousey, R., and Wilson, N., Proc. Rocket Conf. at Oxford (to be published as a supplement of J. Terr. and Atmos. Elect.).

7 Bates, D. R. and Witherspoon, A. E., Mon. Not. Roy. Astro. Soc., 112, 101 (1952).

\section{Polarization of Radar Echoes from Meteorological Precipitation}

Measurements on the polarization of radio waves reflected from meteorological precipitation are sometimes handicapped by limitations of the investigating radar, and this is particularly so in the case of socalled circularly polarized radars. In practice, all radars radiate an elliptically polarized wave, and it can be shown that a perfectly spherical target will appear to produce two orthogonally polarized circular components differing in magnitude by $40 \mathrm{~dB}$. if the voltage ellipticity ratio of the radiated wave is 0.99 , and by $20 \mathrm{~dB}$. if the ellipticity ratio is $0 \cdot 9$. This sets a limit to the accuracy obtainable in field measurements.

A novel type of circularly polarized radar has been constructed, working on a wave-length of $3 \cdot 2 \mathrm{~cm}$., in which particular care has been taken that the polarization parameters are accurately known and controlled 10.99 on transmission and 0.95 on reception), and in addition steps have been taken to eliminate the spurious signal due to departures from circularity in the system. This is achieved by means of a grating in the wave-guide, which separates the incoming signals into two orthogonal circular components; these can be used simultaneously in a bridge system, and may also be combined at inter- 